\title{
Clinical Validation of a Model-based Glycaemic Control Design Approach and Comparison to Other Clinical Protocols
}

\author{
J. Geoffrey Chase, Geoffrey M. Shaw, Christopher E. Hann, Aaron LeCompte, Timothy Lonergan, \\ Michael Willacy, Xing-Wei Wong, Jessica Lin and Thomas Lotz
}

\begin{abstract}
Hyperglycaemia is prevalent in critical care and tight control can reduce mortality from $9-43 \%$ depending on the level of control and the cohort. This research presents a table-based method that varies both insulin dose and nutritional input to achieve tight control. The system mimics a previously validated model-based system, but can be used for long term, large patient number clinical evaluation. This paper evaluates this method in simulation using retrospective data and then compares clinical measurements over 15,000 patient hours to validate the models and development approach. This validation thus also validates the in silico comparison to the landmark clinical tight glycaemic control protocols. Overall, an average clinical glucose level is $5.9 \pm 1.0 \mathrm{mmol} / \mathrm{L}$, matching simulation, however the overall clinical glucose distribution is slightly tighter than that obtained in simulation, indicating that the retrospective virtual trial design approach is slightly conservative. Finally, the model based approach is shown to have tighter control than existing, more ad-hoc clinical approaches based on the simulation results that qualitatively match reported clinical results, but also show significant variation around the average levels obtained in both the hypoand hyper- glycaemic ranges.
\end{abstract}

\section{INTRODUCTION}

Stress induced hyperglycaemia is prevalent in critical care, and can occur in patients with no history of diabetes [1, 2]. Critically ill patients exhibit increased endogenous glucose production, erratic insulin production and increased insulin resistance. Therefore, enteral feeding of glucose and administration of glucocorticoids can further enhance the onset of hyperglycaemia and insulin resistance respectively. Hyperglycaemia also worsens outcomes leading to risk of further complications such as polyneuropathy and multiple organ failure [2]. van den Berghe et al [2, 3] showed that tight glucose control averaging $6.1 \mathrm{mmo} / \mathrm{L}$ reduced mortality up to $45 \%$. Krinsley [4] showed a $17-29 \%$ reduction with a higher glucose average of $7.75 \mathrm{mmol} / \mathrm{L}$.

Model-based protocols for insulin-mediated glucose control in critical care have shown promise, but can have limitations [5]. Due to increased insulin resistance and

Manuscript received April 3, 2006.

J. G. Chase, C. E. Hann, T. Lonergan, A. LeCompte, M. Willacy, X. W. Wong, J. Lin and T. Lotz are with the University of Canterbury, Dept of Mechanical Eng, Centre for Bio-Engineering, Private Bag 4800, Christchurch, New Zealand (+64-3-364-2987 x7224; e-mail: geoff.chase@ canterbury.ac.nz).

G. M. Shaw is with the University of Otago, Christchurch School of Medicine and Health Sciences, Dept of Intensive Care Medicine, Christchurch, New Zealand (e-mail: geoff.shaw@cdhb.govt.nz). insulin effect saturation [6], only limited glycaemic reductions can be made using insulin alone [7]. Hence, the only avenue left to control blood glucose is to also control the exogenous nutritional input that exacerbates stress induced hyperglycaemia [8].

This paper presents the model based development of a robust, table-based protocol ("SPRINT" - Specialized Relative Insulin Nutrition Tables) to maintain blood glucose levels in the target band of 4-6.1 mmol/L [9]. This protocol has been developed based on computerized glycaemic control trials and patient simulations using a physiologically verified insulin-glucose system model. Clinical results are also compared to these simulations to validate the design approach and to compare to other clinical protocols [2-4].

\section{METHODS}

\section{A. System Model}

Tight Glucose control requires capturing the fundamental dynamics of the glucose regulatory system. Chase et al. [57,9] used the system model defined:

$$
\begin{aligned}
& \dot{G}=-p_{G} G-S_{I}\left(G+G_{E}\right) \frac{Q}{1+\alpha_{G} Q}+P(t) \\
& \dot{Q}=-k I+k Q \\
& \dot{I}=-\frac{n I}{1+\alpha_{I} I}+\frac{u_{e X}}{V} \\
& P\left(t_{i}<t<t_{i+1}\right)=\bar{P}_{i+1}+\left(\bar{P}_{i}-\bar{P}_{i+1}\right) e^{-k_{p d}\left(t-t_{i}\right)} \text { where } \bar{P}_{i+1}<\bar{P}_{i} \\
& P\left(t_{i}<t<t_{i+1}\right)=\bar{P}_{i+1}+\left(\bar{P}_{i}-\bar{P}_{i+1}\right) e^{-k_{p r}\left(t-t_{i}\right)} \text { where } \bar{P}_{i+1}>\bar{P}_{i}
\end{aligned}
$$

where $G(t)[\mathrm{mmol} / \mathrm{L}]$ is the plasma glucose above an equilibrium level, $G_{E}[\mathrm{mmol} / \mathrm{L}] . I(t)[\mathrm{mmol} / \mathrm{L}]$ is plasma insulin concentration resulting from exogenous insulin input, $u_{e x}(t)[\mathrm{mU} / \mathrm{min}] . Q(t)[\mathrm{mU} / \mathrm{L}]$ is interstitial insulin concentration and $k[1 / \mathrm{min}]$ accounts for the effective life of insulin in the system. Patient endogenous glucose clearance and insulin sensitivity are $p_{G}[1 / \mathrm{min}]$ and $S_{I}[\mathrm{~L} /(\mathrm{mU} . \mathrm{min})]$, respectively. $V[\mathrm{~L}]$ is the insulin distribution volume and $n$ $[1 / \mathrm{min}]$ is the constant first order decay rate for insulin from plasma. Total plasma glucose input is denoted $P(t)$ $[\mathrm{mmol} /(\mathrm{L} . \mathrm{min})] . k_{p r}$ is the rise rate of rate of plasma glucose input from enterally administered feed [1/min]. $k_{p d}$ is the decay rate of rate of glucose input into plasma from enterally 
administered feed $[1 / \mathrm{min}] . \quad \bar{P}_{i}, \quad \bar{P}_{i+1}$ are stepwise consecutive enteral glucose feed rates [mmol/L.min]. Michaelis-Menten functions are used to model saturation, with $\alpha_{I}[\mathrm{~L} / \mathrm{mU}]$ used for the saturation of plasma insulin disappearance, and $\alpha_{G}[\mathrm{~L} / \mathrm{mU}]$ for the saturation of insulindependent glucose clearance. For the simulations in this study, $k, n, G, I$ and $V$ are set to generic population values [5-7].

Patient specific profiles for time-varying $S_{I}$ and $p_{G}$ can be created by fitting retrospective glucose, insulin and feed data, where is $p_{G}$ accounts for both endogenous clearance and the net impact of endogenous glucose production on removal. This approach is used to create virtual patients [5$7,9]$ to test protocols. Virtual trials use these profiles to determine patient specific blood glucose levels for different insulin and nutrition inputs. Hence, different protocols can be compared for the same patient, a significant advantage in developing and validating new protocols.

The cohort used covers a general cross-section of ICU population, APACHE II score (Average: 21.8, Range: 8-36), age, sex and mortality [7]. Average stay is 3.9 days (Range: 1.4-18.8 days). APACHE II scores are much higher than in $[2,3]$ and [4]. Hence, these patients are more critically ill and more insulin resistant.

\section{B. SPRINT Protocol}

SPRINT is based on a computerized protocol $[5,9,10]$ that regulates both nutritional and insulin inputs. The permissible range of feed variation was $280-700 \mathrm{kcal} /$ day from glucose based on the ideal rate for an $80 \mathrm{~kg}$ male. At the $280 \mathrm{kcal} /$ day minimum, the total caloric intake is still $778 \mathrm{kcal} /$ day [11], which exceeds the level found to avoid an increased risk of bloodstream infections [12].

SPRINT is designed to provide an easy-to-use equivalent to a computerised protocol to enable long term clinical testing of the variable insulin and nutrition control approach. The protocols for controlling feed and insulin inputs were developed through virtual patient trials of the 19-patient cohort to maintain blood glucose levels within the 4$6.1 \mathrm{mmol} / \mathrm{L}$ band. The goal is equal glycaemic control to the computerised method by mimicking its actions $[9,10]$.

The SPRINT protocol consists of two wheels dedicated to enteral nutrition optimisation (specifically RESOURCE ${ }^{\circledR}$ Diabetic) and insulin bolus administration (Actrapid), and is shown in Figure 1. The starting criterion is blood glucose greater than $8.0 \mathrm{mmol} / \mathrm{L}$. The instructions on the "Feed Wheel" in Figure 1 are used to determine the rate of feed as a percentage of the patient's clinically determined goal feed. The result is based on the previous hour's feed level, the current blood glucose concentration and whether blood glucose is rising or falling. The percentage goal feed is converted into an absolute feed rate (in $\mathrm{ml} / \mathrm{hr}$ ) using a patient-specific conversion sticker. The "Insulin Wheel" is then used to determine the insulin bolus size based on the previous insulin bolus size, the current blood glucose level and whether the blood glucose has decreased by more than $1.5 \mathrm{mmol} / \mathrm{L}$. Importantly, the method is effectively fully automated, aside from feed rate, as it relies on no other external clinical inputs or modifications for any patient.
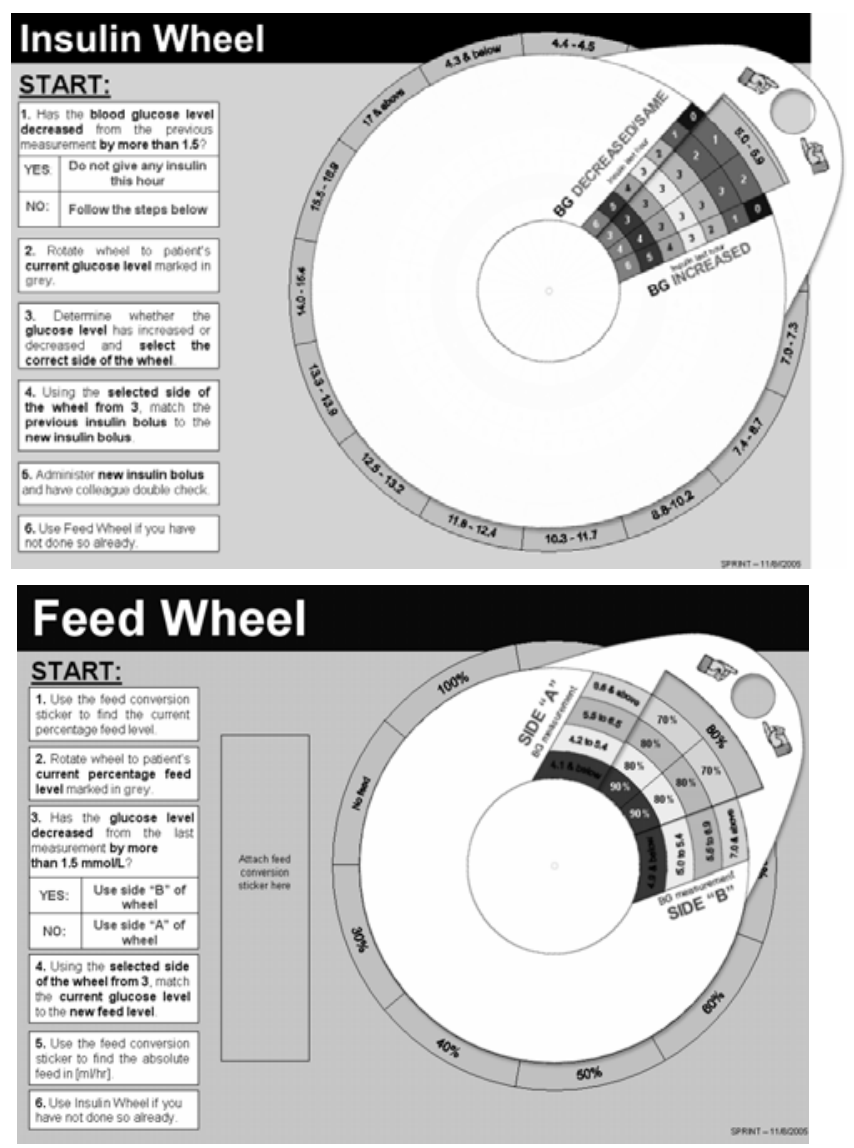

Figure 1: SPRINT insulin and feed wheels [9].

Hourly blood glucose measurements are used to ensure tight control. Two-hourly measurements are used when the patient is stable, defined as 3 consecutive measurements in the $4.0-6.1 \mathrm{mmol} / \mathrm{L}$. For two-hourly measurements, the feed rate is maintained constant, and the same insulin bolus is administered again on the hour between measurements. Two-hourly measurements are continued until the patient leaves the $4.0-6.1 \mathrm{mmol} / \mathrm{L}$ band. SPRINT is stopped when the patient is stable, normoglycaemic, and adequately self regulating. This state is defined as 6 or more hours in the 4$6.1 \mathrm{mmol} / \mathrm{L}$ band, with over $80 \%$ of goal feed rate and a maximum of $2 \mathrm{U} / \mathrm{hr}$ insulin. Finally, insulin is administered via bolus for safety, thus avoiding infusions being left on.

The layout resulted from extensive consultation. Clinical staff reported the system as very easy to use. The covered wheel reduces complexity, reducing error. Overall, SPRINT is simple enough to integrate with any typical ICU practice.

\section{Virtual Trial Protocol Comparison}

Virtual trials are used to compareSPRINT with the published protocols from the landmark studies $[3,4]$. The computerised AIC4 protocol [5] provides another comparison. Protocols that used glucose shots for hypoglycaemia, were modelled at the same value and administered over 5 minutes. No other changes were made from the published protocols. Performance is measured by 
time spent in the $4-6.1 \mathrm{mmol} / \mathrm{L}$ band [3], or the $4-7.75$ $\mathrm{mmol} / \mathrm{L}$ [4] band, rather than a simple average value [13]. Hence, glycaemic levels and the tightness with which they are maintained are both assessed for a more complete analysis of the efficacy.

The simulation approach is Monte Carlo based. Each protocol is run 20 times for all 19 virtual patients including random measurement errors. The results are stored for every glucose measurement the specific protocol required, rather than reporting a morning average or other surrogate. Finally, to validate the comparison and design approach SPRINT clinical results are compared with the simulation results.

\section{ViRTUAL TRIALS RESUlts}

Table 1 shows the virtual trial results for SPRINT, the computerized AIC4 protocol that it mimics, and the two landmark clinical protocols. The clinical protocols are denoted by lead author as "Krinsley" for [4] and "vdB" for [3]. The glucose results in all cases are lognormal $(p<$ 0.005 ), instead of the often assumed normal distribution. The $68.3 \%$ and $95.5 \%$ ranges thus represent 1 and 2 multiplicative standard deviations respectively. The time in band values are percentages of the total trial time. The average insulin and average percentage of goal feed are presented to show the level of interventions.

Table 1: Virtual clinical trial results

\begin{tabular}{|l|c|c|c|c|}
\hline & SPRINT & Krinsley [4] & VdB [3] & AIC4 [5] \\
\hline $50^{\text {th }}$ Percentile & 5.79 & 8.59 & 5.60 & 5.93 \\
\hline Mult. STD & 1.29 & 1.29 & 1.65 & 1.35 \\
\hline $68.3 \%$ range & $(4.5-7.5)$ & $(6.7-11.1)$ & $(3.40-9.24)$ & $(4.4-8.0)$ \\
\hline $95.5 \%$ range & $(3.5-9.6)$ & $(5.2-14.2)$ & $(2.1-15.2)$ & $(3.3-10.8)$ \\
\hline Time in 4-6.1 & $61.7 \%$ & $11.2 \%$ & $35.8 \%$ & $62.2 \%$ \\
\hline Time in 4-7.75 & $83.5 \%$ & $27.4 \%$ & $51.0 \%$ & $82.9 \%$ \\
\hline Time $<4$ & $4.4 \%$ & $0.6 \%$ & $23.6 \%$ & $1.1 \%$ \\
\hline Time $>7.75$ & $12.1 \%$ & $72.0 \%$ & $25.3 \%$ & $16.1 \%$ \\
\hline Avg insulin (U/hr) & 2.4 & 1.6 & 3.0 & 2.6 \\
\hline Avg \% goal feed & $61.9 \%$ & $67.7 \%$ & $67.7 \%$ & $75.8 \%$ \\
\hline
\end{tabular}

Note that the percentage of goal feed values are all less than $100 \%$ as the retrospective data contains clinical stoppages of feed for other clinical causes. The feed levels used are based on the retrospective patients goal feed and the type of enteral or TPN nutrition reported for the given study, which is typically $50 \%$ of calories from dextrose as opposed to the $35 \%$ for the enteral feed used in this clinical study.

The noticeable outlying protocol was from [4], however it is less intensive with a target average of $7.75 \mathrm{mmol} / \mathrm{L}$. The $50^{\text {th }}$ percentile blood glucose levels for SPRINT and AIC4 are comparable with the $[2,3]$. However, the $95.5 \%$ range of 3.50-9.58 $\mathrm{mmol} / \mathrm{L}$, compared to Leuven's to $2.06-15.24$ $\mathrm{mmol} / \mathrm{L}$ from the Leuven study, shows much tighter control, with similar results over the $68.3 \%$ range. Thus, SPRINT and its computerized version (AIC4) tightly regulate blood glucose without significant risk of hypoglycaemia.

The results also compare well with reported average values, suggesting that the computer simulation method produced realistic results. Differences, such as the $2.7 \%$ of measurements below $2.2 \mathrm{mmol} / \mathrm{L}$ in the simulated Leuven protocol compared to $1.0 \%$ in the reported results $[2,3]$, may be due to a more severely ill patient cohort here. Specifically, this virtual cohort has an average APACHE II score of 21.8 versus 9 for the Leuven protocol [2,3] and 16 for the Krinsley protocol [4]. Overall, the results are qualitatively similar to the reported values adding weight to these simulation results.

Another difference may be due to the assumptions made where the protocols referred to specialized clinical input. The insulin dosages recommended by the Leuven protocol were intended as "directives, rather than strict numerical instructions" [3]. Insulin dose adjustments in the Leuven study were also guided by factors, such as body temperature and infection. Retrospective data for these parameters were not available for simulation and the protocol was run on a strict numerical basis, with insulin doses capped at $15 \mathrm{U} / \mathrm{hr}$. The advantage of the AIC4 and SPRINT protocols, in all cases, is that they are essentially fully automated.

\section{Clinical Validation Results}

Figure 2 shows probability density functions for clinical SPRINT results over the first 90 patients and $\sim 13,000$ hours, as compared to the SPRINT and AIC4 simulations for comparison. The clinical cohort has an average APACHE II score of 21 and an average length of stay on SPRINT of $\sim 130$ hours. The similarity of SPRINT and AIC4 is expected as SPRINT mimics the target based, computerized AIC4 controller. Figure 2 also shows simulated results for the van den Berghe et al [2,3] and Krinsley [4] protocols.

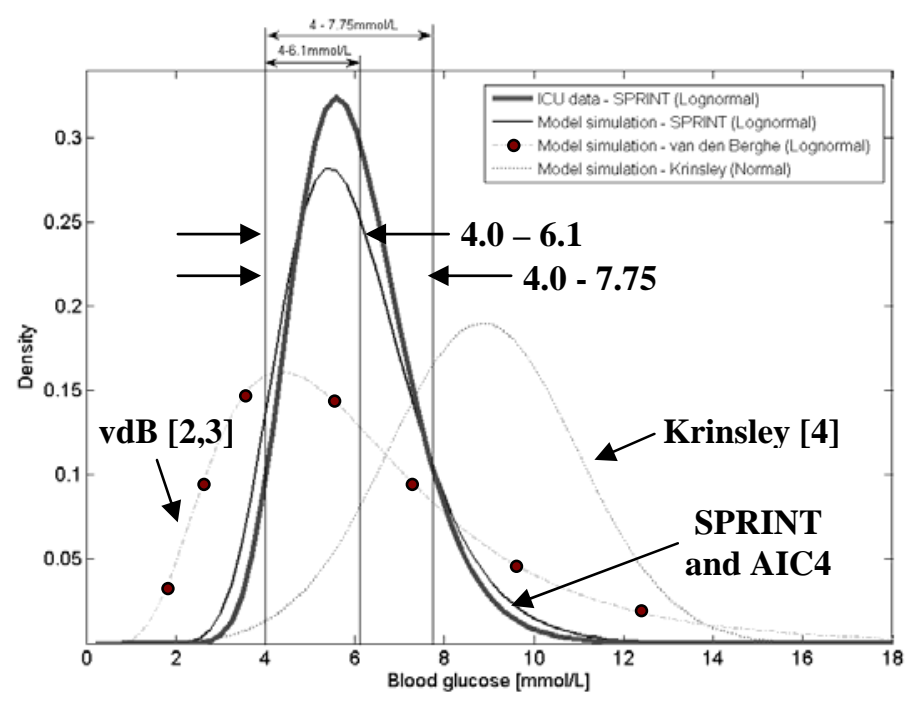

Figure 2: Probability density functions based on both virtual and clinical glycaemic control trial results

Overall, the results in Figure 2 show two main results. 
First, the simulated and clinical SPRINT results are very close, particularly with respect to their width through the 4.0-6.1 mmol/L and 4.0-7.75 mmol/L tight control ranges. In particular, they are tighter through these ranges and overall than the more strongly lognormal results from the protocols used by van den Berghe et al and Krinsley. Second, the simulated and clinical SPRINT results are very close with the gaps representing less than $10 \%$ of all measurements for the simulated results to be outside the clinical results. Hence, comparing simulation and clinical results indicates that the virtual trial simulation approach is both accurate and slightly conservative when deciding to implement them clinically.

In the low and hypo- glycaemic ranges there are significant differences comparing SPRINT results to other protocols. The van den Berghe protocol has $3.5 \%$ of measurements below $2.5 \mathrm{mmol} / \mathrm{L}$ in comparison to $0.1 \%$ for clinical SPRINT results and $0.16 \%$ for virtual trial SPRINT results. Below $4.0 \mathrm{mmol} / \mathrm{L}$, the results are $23.6 \%$ for the van den Berghe protocol versus 2.6\% for clinical SPRINT and $0.6 \%$ for Krinsley's protocol.

With regard to high blood glucose levels there are also significant differences due to the differing levels of tight control achieved. Specifically, $25.3 \%$ of the van den Berghe protocol results are above $7.75 \mathrm{mmol} / \mathrm{L}$ versus $10 \%$ for SPRINT and $70 \%$ for the more conservative protocol used by Krinsley. Note that of the $10 \%$ above $7.75 \mathrm{mmol} / \mathrm{L}$ from SPRINT $2 \%$ occur in the first $12-24$ hours of use due as the initially elevated blood glucose levels are reduced.

\section{DISCUSSION}

The variable nutrition and insulin approach presented is shown to be highly effective at tightly controlling blood glucose levels. Both of these protocols were developed using the virtual trial method and the virtual patient cohort presented in this work. The SPRINT table based version and the computerized AIC4 method it mimics also deliver very similar results, further validating the approach.

The overall data analysis presented is statistically valid, however there may be some differences in the protocols modeled for the Leuven and Krinsley protocols. Differences are largely due to the lack of complete published protocol information. In particular, both protocols use additional data and can rely on unspecified, patient specific clinical modification of treatment that is not reported.

In contrast, the SPRINT and AIC4 protocols are effectively fully automated. In all clinical studies, neither SPRINT nor the AIC4 protocol required any clinical modification. Thus, the only patient specific aspect is the goal feed level $[5,9,10]$ and protocols are otherwise used identically for all patients, unlike other published results.

\section{Conclusions}

There are two main results from this work. First, the virtual patient simulation and design approach is presented and validated as an effective means of developing clinical protocols for tight glycaemic control. The results when implemented clinically are shown to be, for the cases presented, very close to reported clinical results, with differences attributable to differences in patient cohort and level of critical illness. Second, the variable insulin and nutrition approach, as seen with the SPRINT protocol, provides a long term and stable method of tightly controlling blood glucose for a very critically ill cohort and over very long periods of stay. Finally, the approach is seen to be slightly conservative in making clinical implementation decisions from the simulated virtual trials.

\section{REFERENCES}

[1] S. E. Capes, D. Hunt, K. Malmberg, and H. C. Gerstein, "Stress hyperglycaemia and increased risk of death after myocardial infarction in patients with and without diabetes: a systematic overview," Lancet, vol. 355, pp. 773-778, 2000.

[2] G. Van den Berghe, P. Wouters, F. Weekers, C. Verwaest, F. Bruyninckx, M. Schetz, D. Vlasselaers, P. Ferdinande, P. Lauwers, and R. Bouillon, "Intensive insulin therapy in the critically ill patients," $N$ Engl J Med, vol. 345, pp. 1359-1367, 2001.

[3] G. Van den Berghe, P. J. Wouters, R. Bouillon, F. Weekers, C. Verwaest, M. Schetz, et al, "Outcome benefit of intensive insulin therapy in the critically ill: Insulin dose versus glycemic control," Crit Care Med, vol. 31, pp. 359-366, 2003.

[4] J. S. Krinsley, "Decreased mortality of critically ill patients with the use of an intensive glycemic management protocol," Crit Care Med, vol. 31, pp. A19, 2003.

[5] XW Wong, GM Shaw, CE Hann, T Lotz, J Lin, I Singh-Levett, L Hollingsworth, OS Wong and JG Chase. "Optimised Insulin and Nutrition Delivery via Model Predictive Control for Tight Glycaemic Regulation in Critical Care," Diabetes Technology \& Therapeutics (DT\&T), Vol 8(2), pp. 174-190, 2006.

[6] JG Chase, GM Shaw, J Lin, CV Doran, M Bloomfield, GC Wake, B Broughton, CE Hann, and T Lotz, "Impact of Insulin-Stimulated Glucose Removal Saturation on Dynamic Control of Hyperglycaemia," J Intell Systems Tech and Appl, Vol. 1, pp. 79-94, 2004.

[7] C. E. Hann, J. G. Chase, J. Lin, T. Lotz, C. V. Doran, and G. M. Shaw, "Integral-based parameter identification for long-term dynamic verification of a glucose-insulin system model," Comp Methods Prog Biomed, vol. 77, pp. 259-70, 2005.

[8] J. F. Patino, S. E. de Pimiento, A. Vergara, P. Savino, M. Rodriguez, and J. Escallon, "Hypocaloric support in the critically ill," World J Surg, vol. 23, pp. 553-9, 1999.

[9] T Lonergan, A LeCompte, M Willacy, JG Chase, GM Shaw, XW Wong, T Lotz, J Lin, and CE Hann. "A Simple Insulin-Nutrition Protocol for Tight Glycemic Control in Critical Illness: Development and Protocol Comparison," Diabetes Technology \& Therapeutics (DT\&T), Vol 8(2), pp. 191-206, 2006.

[10] X. W. Wong, J. G. Chase, G. M. Shaw, C. Hann, J. Lin, and T. Lotz, "Comparison of adaptive and sliding scale glycaemic control in critical care and the impact of nutritional inputs," presented at 12th Intl Conf on Biomedical Eng (ICBME), 2005.

[11] Novartis, "RESOURCE® Diabetic Nutrition Information." US: Novartis Med Nutrition, 2005.

[12] L. Rubinson, G. B. Diette, X. Song, R. G. Brower, and J. A. Krishnan, "Low caloric intake is associated with nosocomial bloodstream infections in patients in the medical intensive care unit," Crit Care Med, vol. 32, pp. 350-7, 2004.

[13] Chase, JG, Shaw, GM, Wong, XW, Lotz, T, Lin, J and Hann, CE "Model-based Glycaemic Control in Critical Care - A review of the state of the possible," Biomedical Signal Processing \& Control (BSPC), in-press, 2006. 